OPEN ACCESS

Edited by:

Jianxun Ding,

Chinese Academy of Sciences, China

Reviewed by:

Da Huang,

Fuzhou University, China

Wenguo Cui,

Shanghai Jiao Tong University, China

Zhitao Gao,

Xinxiang Medical University, China

*Correspondence:

Lining Zhang

zhangln301@163.com

Yilan Li

liyilan1367@163.com

${ }^{\dagger}$ These authors have contributed equally to this work

Specialty section:

This article was submitted to

Biomaterials,

a section of the journal

Frontiers in Bioengineering and

Biotechnology

Received: 06 August 2020

Accepted: 12 October 2020

Published: 27 November 2020

Citation:

Wang J, Wang L, Gao Y, Zhang Z, Huang $X$, Han T, Liu B, Zhang Y, Li Y

and Zhang $L$ (2020) Synergistic

Therapy of Celecoxib-Loaded Magnetism-Responsive Hydrogel

for Tendon Tissue Injuries.

Front. Bioeng. Biotechnol. 8:592068.

doi: 10.3389/fbioe.2020.592068

\section{Synergistic Therapy of Celecoxib-Loaded \\ Magnetism-Responsive Hydrogel for Tendon Tissue Injuries}

\author{
Jingxin Wang ${ }^{1 t}$, Likang Wang ${ }^{2 t}$, Yueming Gao ${ }^{3}$, Zhao Zhang ${ }^{4}$, Xiaofeng Huang ${ }^{5}$, \\ Tong Han', Biyuan Liu' ${ }^{1}$, Yujie Zhang ${ }^{6}$, Yilan Li' ${ }^{1 *}$ and Lining Zhang ${ }^{7 *}$

\begin{abstract}
${ }^{1}$ Department of Rehabilitation, Zhengzhou Central Hospital Affiliated to Zhengzhou University, Zhengzhou, China, ${ }^{2}$ Department of Rehabilitation Medicine, The Third Medical Centre, Chinese PLA General Hospital, Beijing, China, ${ }^{3}$ Department of Rehabilitation Medicine, The Second Medical Centre, Chinese PLA General Hospital, Beijing, China, ${ }^{4}$ Graduate School, Chinese PLA General Hospital, Beijing, China, ${ }^{5}$ Department of Endocrinology, The Second Medical Centre, Chinese PLA General Hospital, Beijing, China, ${ }^{6}$ Department of Epidemiology, School of Public Health Southern Medical University, Guangzhou, China, ${ }^{7}$ Department of Rehabilitation Medicine, The First Medical Centre, Chinese PLA
\end{abstract} \\ General Hospital, Beijing, China
}

Tendon tissue injury is very common and always associated with pain, tissue swelling and even malformation if not treated on time. Traditional therapeutic strategies, such as cryotherapy, electrical therapy, ultrasound therapy and anti-inflammatory drug, are still unsatisfying. In this work, a synergistic therapy, based on the combination of celecoxib drug and pulsed electromagnetic field (PEMF) regimens, was developed for the treatment of tendon injury. This celecoxib-loaded magnetism-responsive hydrogel dressing (gelatin/ $/ \mathrm{Fe}_{3} \mathrm{O}_{4} /$ celecoxib) showed good biocompatibility and coordinated drug release behavior under the PEMF, which could effectively reduce the inflammatory reaction of macrophage cells with the incremental proportion of M2 macrophages at the injury site. CatWalk gait analysis further verified this synergistic effect of combination therapy for achieving the outstanding recovery of the injured tendon tissue. Thus, this magnetism-responsive hydrogel may represent a promising alternative strategy in clinics for promoting tendon healing.

Keywords: tendon injury, celecoxib, macrophages, pulsed electromagnetic field, gait analysis

\section{INTRODUCTION}

Tendon injury is a common disorder that cause pain and motor dysfunction, which is one of the top 15 diseases with the highest incidence according to the statistics of the American Health System Diseases (Global Burden of Disease Study 2013 Collaborators, 2015). On account of the tendon pain, chronic degeneration or even fracture, it requires high cost of surgery and rehabilitation of 13,000-20,000 dollars per case with the long treatment period, thus seriously affecting the life 
qualities and activities of sufferers (Carr et al., 2015; Voleti et al., 2012). Even so, the newborn scar tissues after the tendon repair still display the abnormal structures, poor mechanical properties and high incidence of fracture after operation (Biressi et al., 2014), which brings about a huge economic burden to both families and society. Therefore, to thoroughly analyze the reasons in depth, effectively promote tendon healing and improve limb functions are great challenges for orthopedics and rehabilitation physicians.

Clinical studies show that inflammation exists during the repair of tendon injuries. A large number of inflammatory cells (macrophages and mast cells) were found in biopsies of human calcified tendinitis (Hackett et al., 2016). The biggest difference between adult tendon repair and embryonic tendon repair is the presence of an inflammatory response (Tang et al., 2014). Embryonic tendon repair is a regeneration mode without scar hyperplasia. Embryonic fibroblasts can reduce the recruitment of inflammatory cells to the injury site, and adult tendon repair belongs to scar healing. Tendon healing period includes the inflammatory, proliferation and remodeling processes. The main inflammatory cells in the process of tendon repair are macrophages with M1 type and M2 type (Voleti et al., 2012; Martinez and Gordon, 2014). M1 macrophages mainly exist in the early inflammatory time of tendon repair, which are beneficial for removing necrotic tissue and initiating tendon repair. However, it secretes pro-inflammatory cytokines of IL1 , TNF- $\alpha$, and IL- 6 that can induce the inflammation, scar formation, cells death and matrix degeneration to hinder the tendon repair. While M2 macrophages mainly exist in the late remodeling stage and produce the anti-inflammatory factor of IL-1 receptor antibodies (IL-1RA), IL-10, growth factors and extracellular matrix, which can effectively reduce inflammation and scars to promote the matrix remodeling and wound healing (Linderman et al., 2016).

Macrophage phenotype changes play an important role from the initial stage of inflammatory response to the fibrosis and remodeling stage of tendon injury (Sugg et al., 2014), and the macrophage infiltration and activation are initiating factors of tissue fibrosis. Previous studies have shown that the number of infiltrating macrophages is directly proportional to the degree of tissue fibrosis (Duffield, 2010). In the regulatory mechanism, the Janus kinase/signal transducer and activator of tran-ions (JAK/STAT) is the core pathway for inducing the macrophage activation and participating in the process of excessive fibrosis or remodeling after liver, kidney, cardiovascular and other tissue damages (Gombozhapova et al., 2017). Recently, the expression of STAT- 6 was found in biopsy tissues of patients with advanced supraspinatus tendinitis, exhibiting the potential relation to the relief of tendon pain (Dakin et al., 2015), which indicated that the JAK/STAT-6 signaling pathway may be involved in macrophages tendon tissue and played an important role in the process of remodeling after injury, however, the role of JAK/STAT signaling pathway-mediated inflammation regulation in tendon remodeling was unclear so far as well as the regulation effects of cell polarization on the tendon remodeling after injury.

In the clinical work, many scholars try to use non-steroidal, anti-inflammatory and sugar cortical hormone drugs to inhibit the tendon disease inflammation. For example, celecoxib is the non-steroidal anti-inflammatory drug (COX-2 inhibitor) to inhibit plasma exudation causing inflammatory edema, which have shown that celecoxib inhibits the expression of inflammatory genes such as COX-2, NO, IL-6, MIP- $1 \alpha$, IL-1 $\beta$ through TLR2, JNK, and NF- $\kappa \mathrm{B}$, thus possessing an effective analgesic and anti-inflammatory activity in the treatment of tendon injury. However, two main disadvantages of conventional celecoxib are low concentration after oral administration and rapid drug clearance from the wound, which necessitates frequent large doses for maintaining effective drug concentration but with inevitable side effects. In addition, oral non-steroidal antiinflammatory drugs are the prevailing chronic treatment option for tendon injury, however, their prolonged use is controversial due to their high risk to benefit ratio. Therefore, formulation of drugs in a controlled delivery system is of paramount importance to allow for prolonged drug residence time when long term treatment is required.

Hydrogels are an attracting "soft-wet" material that consists plenty of water and distinctive 3D cross-linked polymeric network structures, which has aroused extensive attention due to its broad applications ranging from food chemistry and cosmetics to medical implants and scaffolds for tissue engineering (Liu H.Y.et al., 2020; Tang et al., 2020; Liu B.C.et al., 2020; Chen et al., 2019; Wang et al., 2020; Bao et al., 2020; Yang et al., 2021). Collagen, extracted from animals, is the main component of the extracellular matrix in mammalian tissues, such as skin, bone, cartilage, tendons and ligaments, which have been one of the most widely used natural hydrogellike substances. Gelatin is a derivative of collagen and formed by breaking down the triple helix structure of collagen into single-stranded molecules, possessing no adverse reactions, no immunogenicity and good biodegradability in vivo. Therefore, gelatin hydrogels can be used as drug carriers to be applied or transplanted into an organism, thereby providing therapeutic benefits for achieving the controlled drug release system. Pulsed electromagnetic field therapy (PEMF) has already been a clinical method to accelerate tissue healing and recovery. Researches have suggested that PEMF could reduce pain via its effect on nitric oxide, calmodulin and/or opioid pathways, and it's also a potential method to promote the drug absorption and tendon repair (Wanitphakdeedecha et al., 2017). In addition, pulsed electromagnetic field can change osmotic pressure and permeability, accelerate the blood circulation, reduce the colloid osmotic pressure of tissues, promote the exudate absorption, facilitate the protein transfer and relieve swelling and pain, which has a good therapeutic effect on the bloody and inflammatory swelling. In this work, we prepared a hydrated hydrogel dressing (gelatin $/ \mathrm{Fe}_{3} \mathrm{O}_{4} /$ celecoxib) with the synergistic therapies of celecoxib drugs and PEMF for the effective treatment of tendon tissue injury (Scheme 1). After tuning the pulsed electromagnetic field, the directional movement of embedded $\mathrm{Fe}_{3} \mathrm{O}_{4}$ nanoparticles can accelerate the celecoxib release, and meanwhile the generated heat can also loosen the gelatin network structures and further promote the drug release rate, exhibiting a kind of coordinated drug release behavior. In addition, in vivo implanting experiments were carried out to verify the biocompatibility and synergistic 

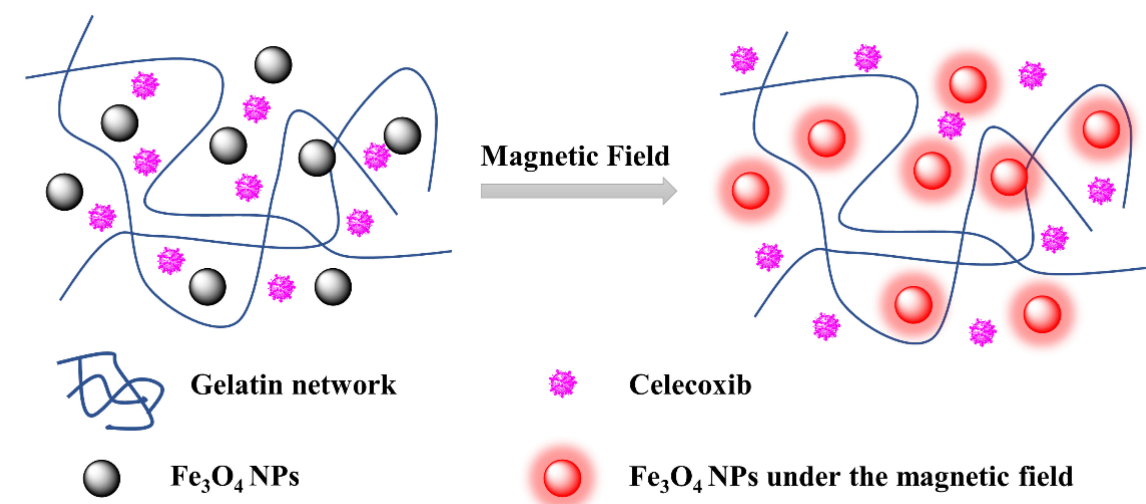

Celecoxib

$\mathrm{Fe}_{3} \mathrm{O}_{4}$ NPs under the magnetic field

SCHEME 1 | Schematic for formation of gelatin/ $/ \mathrm{Fe}_{3} \mathrm{O}_{4} /$ celecoxib system for the synergetic therapy of tendon tissue injuries.

therapeutic effect. Combined with gait analysis, it was powerfully revealed that this synergistic therapy was significantly crucial and effectively used for tendon injuries repair and reshaping in clinical applications.

\section{EXPERIMENTAL SECTION}

\section{Materials}

Gelatin, ferric acetylacetonate $\left(\mathrm{Fe}(\mathrm{acac})_{3}\right)$, 1,2-hexadecanediol and polyol medium triethylene glycol were purchased from J\&K. Celecoxib was purchased from Dalian Meilun Biotechnology Co., LTD. All chemicals were used as received. All other chemicals were of analytical grade.

\section{Animals}

Sprague Dawley rats $(200 \mathrm{~g})$ were purchased from the Animal Center of the Chinese PLA General Hospital. The animals were acclimatized for 1 week in an animal facility under controlled conditions of temperature $\left(23 \pm 3^{\circ} \mathrm{C}\right)$, relative humidity $(55 \pm 10 \%)$ and light $(12 / 12 \mathrm{~h}$ light/dark, with no ultraviolet exposure). The animals had free access to a laboratory diet and ion-sterilized tap water. All experiments were performed in accordance with the guidelines of the care and use of laboratory animals of the Chinese PLA General Hospital, and experiments were approved by the animal ethics committee of the Chinese PLA General Hospital.

\section{Synthesis of Water-Soluble $\mathrm{Fe}_{3} \mathrm{O}_{4} \mathrm{NPs}$}

$\mathrm{Fe}(\mathrm{acac})_{3}$ (1 mmol, 99\%, Acros) and polyol medium triethylene glycol $(30 \mathrm{~mL})$ were mixed together and slowly heated to reflux $\left(278^{\circ} \mathrm{C}\right)$ for 30 min under argon protection, producing a black homogeneous colloidal suspension. After cooled down to room temperature, $20 \mathrm{~mL}$ of ethyl acetate was added to the reaction solution, resulting in a black precipitation of magnetite nanoparticles which was then separated from the solution by a pulsed electromagnetic field. After being washed by ethyl acetate for three times, the precipitation was re-dispersed in water for further use.

\section{Preparation of the Gelatin/ $\mathrm{Fe}_{3} \mathrm{O}_{4} /$ Celecoxib Hydrogel}

Firstly, gelatin was added into $10 \mathrm{~mL}$ of PBS solutions and heated to $40^{\circ} \mathrm{C}$ in an oil bath to form a transparent agarose solution, then celecoxib and $\mathrm{Fe}_{3} \mathrm{O}_{4}$ NPs were added into the solution. After cooling down to the room temperature, gelatin $/ \mathrm{Fe}_{3} \mathrm{O}_{4} /$ celecoxib hydrogel was formed in the suitable molds.

\section{Scanning Electron Microscopy (SEM)}

The hydrogels were prepared as described above and then freeze-dried at $-50^{\circ} \mathrm{C}$ for $48 \mathrm{~h}$. Then the samples were carefully stuck onto the conducting resin with double-sided adhesive, and sputter-coated with a thin layer of $\mathrm{Pt}$ for $90 \mathrm{~s}$ to make the sample conductive before testing. SEM images were obtained at acceleration voltage of $5 \mathrm{kV}$ on a JSM-6700F microscope (JEOL, Japan).

\section{In vitro Drug Release}

Only one side of the hydrogel would touch the tissue during drug release process, so we employed the same release model as reported previously (Li et al., 2020). The hydrogel was prepared in a container with the diameter of $10 \mathrm{~mm}$ and height of $2 \mathrm{~mm}$ while the celecoxib was encapsulated inside the hydrogel. Then, the drug-contained hydrogel was immersed into the PBS $(\mathrm{pH}$ 7.4) and the solutions were collected at special intervals of time. During each interval, the magnetic field (pulsed magnetic therapy apparatus, 301-M9) was applied for $30 \mathrm{~min}$. The collected solution at different time were tested using the HPLC system. The release without the PEMF was set as control.

\section{In vitro Cytotoxicity}

In vitro cytotoxicity assays of gelatin/celecoxib and gelatin/ $/ \mathrm{Fe}_{3} \mathrm{O}_{4}$ hydrogel were evaluated through the mouse embryos osteoblast precursor cells (MC3T3-E1) by the CCK-8 assay. Firstly, extraction of the hydrogel was obtained by immersing the hydrogel into the $1 \mathrm{~mL}$ of Dulbecco's modified Eagle medium (DMEM). The MC3T3-E1 mouse fibroblasts were plated in 96well cell culture at a density of $10^{4}$ cells/well, incubated with the extraction under $5 \% \mathrm{CO}_{2}$ incubator at $37^{\circ} \mathrm{C}$ for 1 and 3 days and 
then changed to $100 \mu \mathrm{L}$ of fresh DMEM and $10 \mu \mathrm{L}$ of CCK8. Subsequently, the cells were incubated at $37^{\circ} \mathrm{C}$ for another $4 \mathrm{~h}$ in a $\mathrm{CO}_{2}$ incubator. Then the cell viability was evaluated by comparing the absorbance of measured solutions using microplate reader at $450 \mathrm{~nm}$. The final results were assumed to be the means of triplicate.

\section{Confocal Laser Scanning Microscopy (CLSM) Observation}

MC3T3-E1 cells were seeded into 24-well culture plates at a density of 8,000 cells per well and incubated for $24 \mathrm{~h}$ at $37^{\circ} \mathrm{C}$ in humidified air containing 5\% $\mathrm{CO}_{2}$. By using complete DMEM containing $10 \% \mathrm{FBS}, 50 \mathrm{IU} \mathrm{mL}^{-1}$ penicillin and $50 \mathrm{IU} \mathrm{mL}^{-1}$ streptomycin, the culture media were replaced by extraction of the hydrogel. After cultivating for prescribed time intervals, MC3T3-E1 cells were washed by cold buffer solution for three times and immersed with $4 \%$ paraformaldehyde solution for $0.5 \mathrm{~h}$ at room temperature to fix the cell configuration. Then 4,6diamidino-2-phenylindole (DAPI) was used for cellular staining and the process continued for $20 \mathrm{~min}$. Finally, MC3T3-E1 cells were washed by buffer solution and directly transferred to glass culture dish for CLSM observation.

\section{Animal Model of Achilles Tendon Rupture}

After the experimental animals were stabilized for 1 week, fifty SD rats were randomly divided into five groups of control (not treated), model (placebo treated), gelatin/celecoxib, gelatin/ $\mathrm{Fe}_{3} \mathrm{O}_{4}$ and gelatin/ $\mathrm{Fe}_{3} \mathrm{O}_{4} /$ celecoxib hydrogel, which were anesthetized with an intravenous injection of pentobarbital sodium $(50 \mathrm{mg} / \mathrm{kg})$ and sterile skin preparation. Then a transverse incision was made at $0.5 \mathrm{~cm}$ of the insertion point in the Achilles tendon of rats. During the operation, the skin was cut and attention was paid to the protection of blood vessels to prevent vascular damage after the lumen, exposing the Achilles tendon tissue. Finally, a 1-2 mm of transverse defect was made to establish the rupture model of the Achilles tendon. After treatment of the Achilles tendon, the wound was washed with normal saline. The broken end of the Achilles tendon was not treated, and the skin was sutured with needle and thread followed by intramuscular injection of penicillin $1 \times 10^{5} \mathrm{U} \mathrm{d}^{-1}$ for three continuous days to prevent infection. Then, the injured rats were treated by the hydrogel dressing with and without the PEMF $(0.3$ T) and celecoxib. After treatment for 3, 7, 14, and 28 days, the rats were euthanized and the tendon tissues of rats were collected for $\mathrm{H} \& \mathrm{E}$ staining staining for the observation and analysis of daily activities, gait and wound healing.

\section{Gait Analysis Using CatWalkTM}

The CatWalkTM system was used to perform a detailed analysis of gait. In brief, the rats were placed in the system on a glass plate in a darkened room and allowed to walk freely. Light beams from a fluorescent lamp were sent through the glass plate, and the light beams were completely reflected internally. When a paw touched the glass plate, the light beams were reflected downward.

Thirty-five rats were randomly divided into five groups according to the random number table method: control, model, gelatin $/ \mathrm{Fe}_{3} \mathrm{O}_{4}$, gelatin/celecoxib and gelatin $/ \mathrm{Fe}_{3} \mathrm{O}_{4} /$ celecoxib, and the rats were treated with samples at the rupture of Achilles tendon. In our experiment, the gait patterns of seven rats from each group were recorded with three times and analyzed automatically using the CatWalk system. Gait patterns analyzed included standing phase time, swing phase time, footprints area, mean intensity and swing speed. After observation for 1, 3, 7, 14, and 28 days, the gait parameters were measured and selected for analysis with a CatWalk gait analyzer.

\section{Statistical Analysis}

All quantitative data were statistically analyzed via the $t$-test. $P$-value $(<0.05)$ was considered statistically significant for all analyses.

\section{RESULTS AND DISCUSSION}

\section{Characterization of Water-Soluble $\mathrm{Fe}_{3} \mathrm{O}_{4}$ NPs}

Pulsed electromagnetic field therapy has become a clinical method to accelerate tissue healing and recovery. To combine magnet therapy with drug therapy, we firstly prepared watersoluble $\mathrm{Fe}_{3} \mathrm{O}_{4}$ nanoparticles (NPs) with the size of ca. $10 \mathrm{~nm}$ to provide an external magnetic field targeting function (Zhang et al., 2019). After the simple sol-gel transition process, we can easily obtain the transparent gelatin in Figure 1A. On account of the poor water solubility of celecoxib, gelatin/celecoxib hydrogel was white and opaque (Figure 1B) while the gelatin $/ \mathrm{Fe}_{3} \mathrm{O}_{4}$ hydrogel was black and dense (Figure 1C). Therefore, black gray and opaque state in Figure 1D demonstrate the successful encapsulation of magnetic nanoparticles and drug molecules within the gelatin $/ \mathrm{Fe}_{3} \mathrm{O}_{4} /$ celecoxib hydrogel.

\section{Preparation of Drug-Loaded Magnetism-Responsive Composite Hydrogels}

As shown in Figure 2, gelatin/ $\mathrm{Fe}_{3} \mathrm{O}_{4} /$ celecoxib hydrogels possessed porous structures with a dimension of ca. $60 \mu \mathrm{m}$, which was benefited for the nutrient exchange and drug delivery. Since the $\mathrm{Fe}_{3} \mathrm{O}_{4}$ NPs and celecoxib drugs were incorporated into the hydrogel in situ, we could observe some aggregates of the $\mathrm{Fe}_{3} \mathrm{O}_{4}$ and the celecoxib nanoparticles within the networks. In addition, the regular network structures indicated the favorable mechanics that will be feasible for the operative procedures.

\section{In vitro Drug Release}

For synergistic effect of treatment, drugs were incorporated, of which the release can be controlled by extra magnetic field. Under the PEMF, the shaking of $\mathrm{Fe}_{3} \mathrm{O}_{4}$ NPs in the network could loosen the network to increase drug release rate, and the effect would be enhanced when the hydrogel was porous. In addition, heat can be generated to further accelerate the drug release (Xu et al., 2015; Liu Z.Y.et al., 2020). Considering 

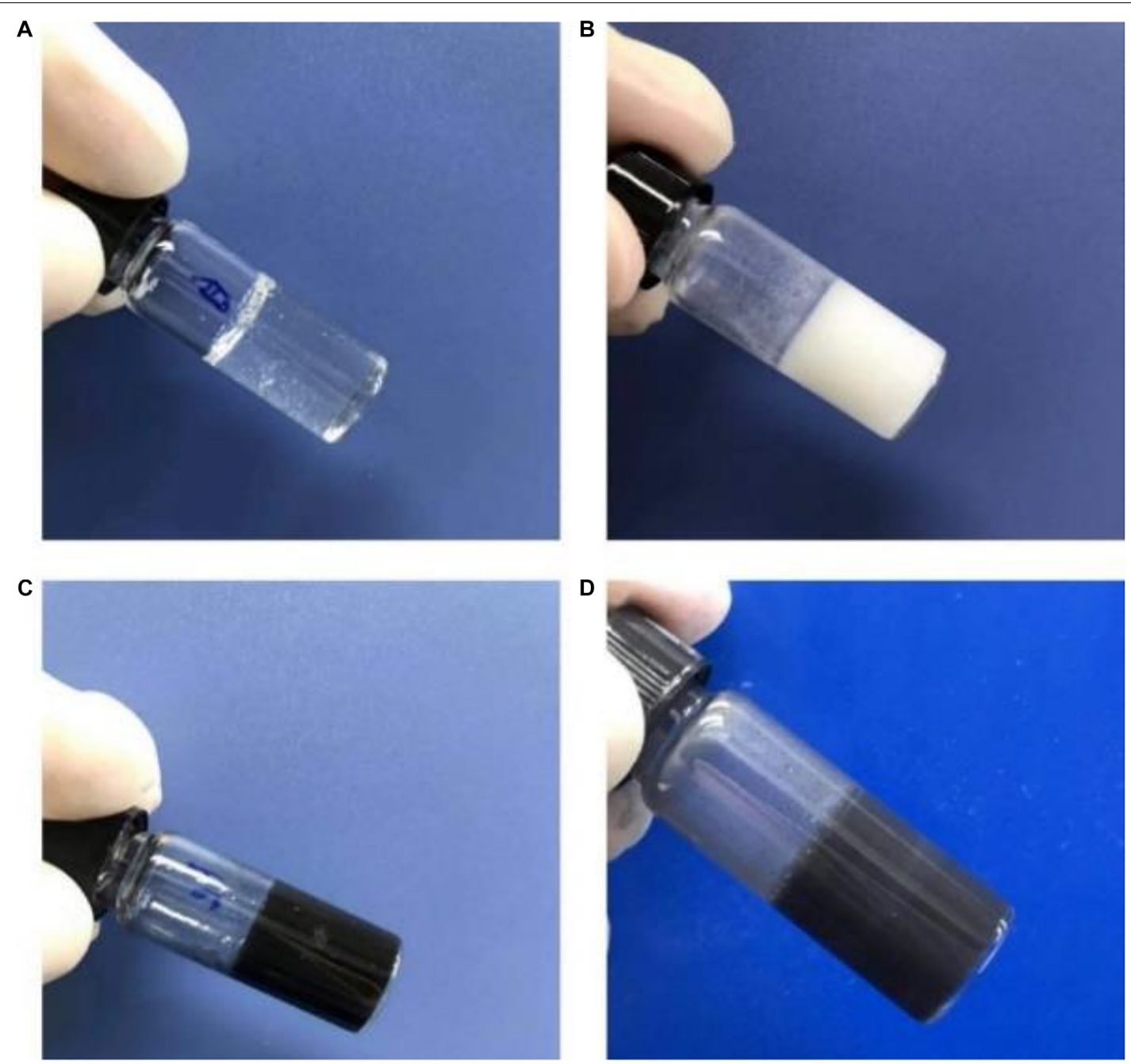

FIGURE 1 | Photos of (A) gelatin, (B) gelatin/celecoxib hydrogel, (C) gelatin/ $\mathrm{Fe}_{3} \mathrm{O}_{4}$ hydrogel and (D) gelatin/Fe $3 \mathrm{O}_{4} / \mathrm{celecoxib}$ hydrogel.

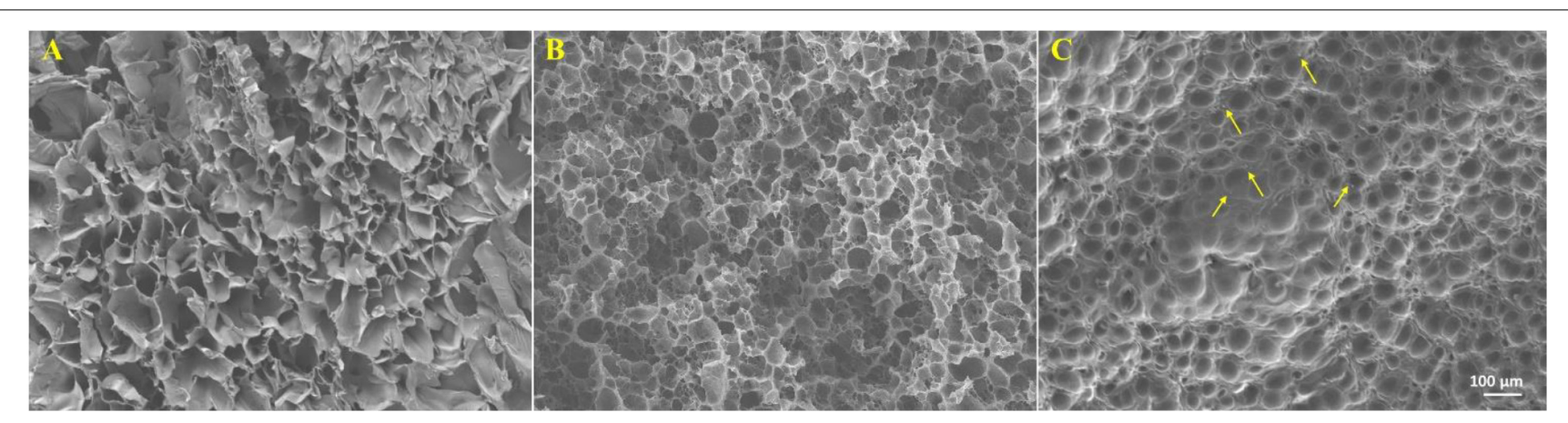

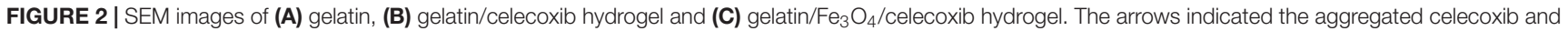
$\mathrm{Fe}_{3} \mathrm{O}_{4} \mathrm{NPS}$.

that only one side of the hydrogel would contact the injured tendon tissue, gelatin $/ \mathrm{Fe}_{3} \mathrm{O}_{4} /$ celecoxib hydrogel was put in the receptacle with the diameter of $10 \mathrm{~mm}$ and height of $2 \mathrm{~mm}$ so that only one side was accessible to the release medium
(Figure 3A). HPLC was used to record the release medium of celecoxib at different time. Figure 3B showed that the release speed under the magnet field was higher, and the total release amount under the pulsed electromagnetic field was $75.2 \pm 3.5 \%$ 

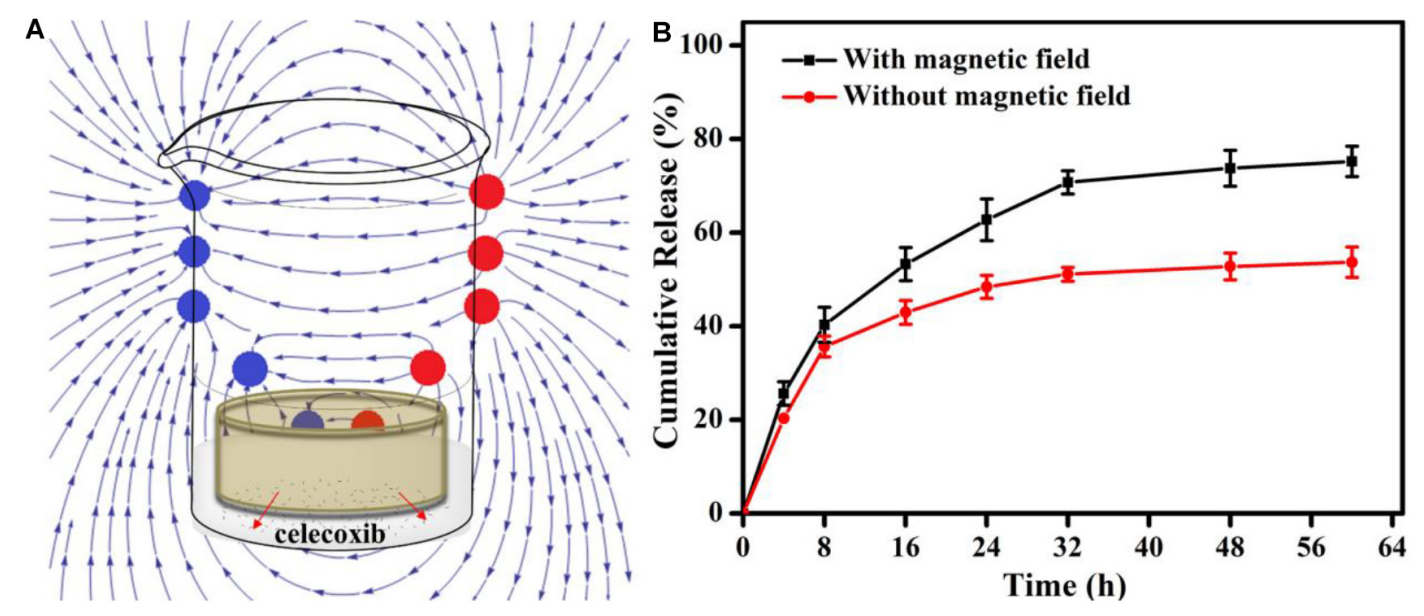

FIGURE 3 | (A) Schematic showing the in vitro release experiment under the PEMF. (B) The release profiles of the dressing with (red) or without (black) the PEMF.

A
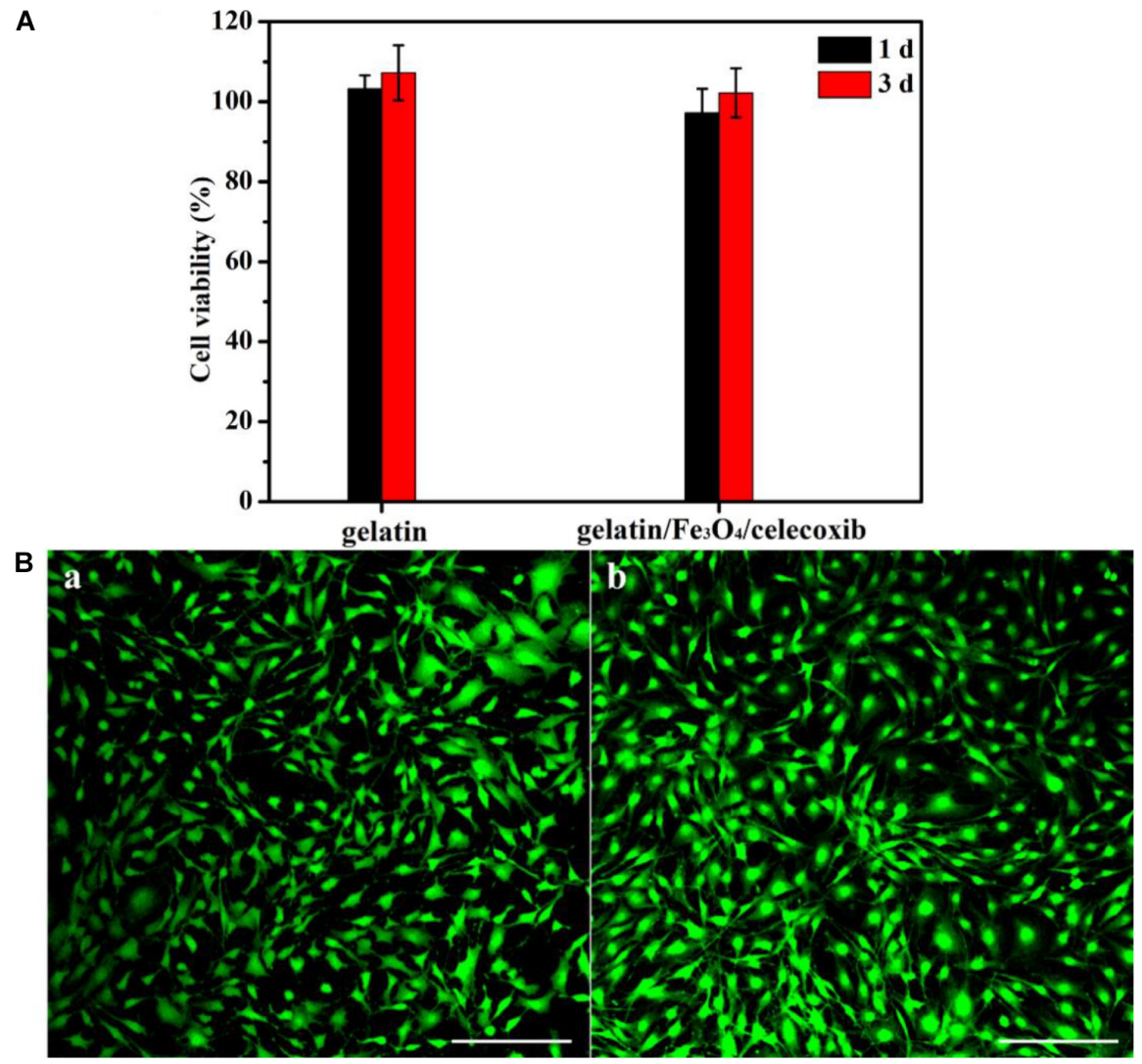

FIGURE 4 | (A) Cell viability of gelation and gelatin/ $\mathrm{Fe}_{3} \mathrm{O}_{4} / \mathrm{celecoxib}$ hydrogels to MC3T3-E1 cells. (B) CLSM images of (a) gelatin and (b) gelatin/Fe $\mathrm{O}_{4} / \mathrm{celecoxib}$ hydrogel in MC3T3-E1 cells following $24 \mathrm{~h}$ incubation.

compared to $53.7 \pm 3.2 \%$ without magnetic field. Under the effect of pulsed electromagnetic field, the directional movement of embedded $\mathrm{Fe}_{3} \mathrm{O}_{4}$ nanoparticles can accelerate the celecoxib release, and meanwhile the generated heat can also loosen the gelatin network structures and further promote the drug release rate, presenting a coordinated drug release behavior and providing the possibility to combine the magnetic therapy and drug therapy for tendon repair.

\section{In vitro Biocompatibility}

The in vitro cytotoxicity of gelation and gelatin $/ \mathrm{Fe}_{3} \mathrm{O}_{4} /$ celecoxib hydrogels was evaluated by CCK- 8 assay. After incubation 


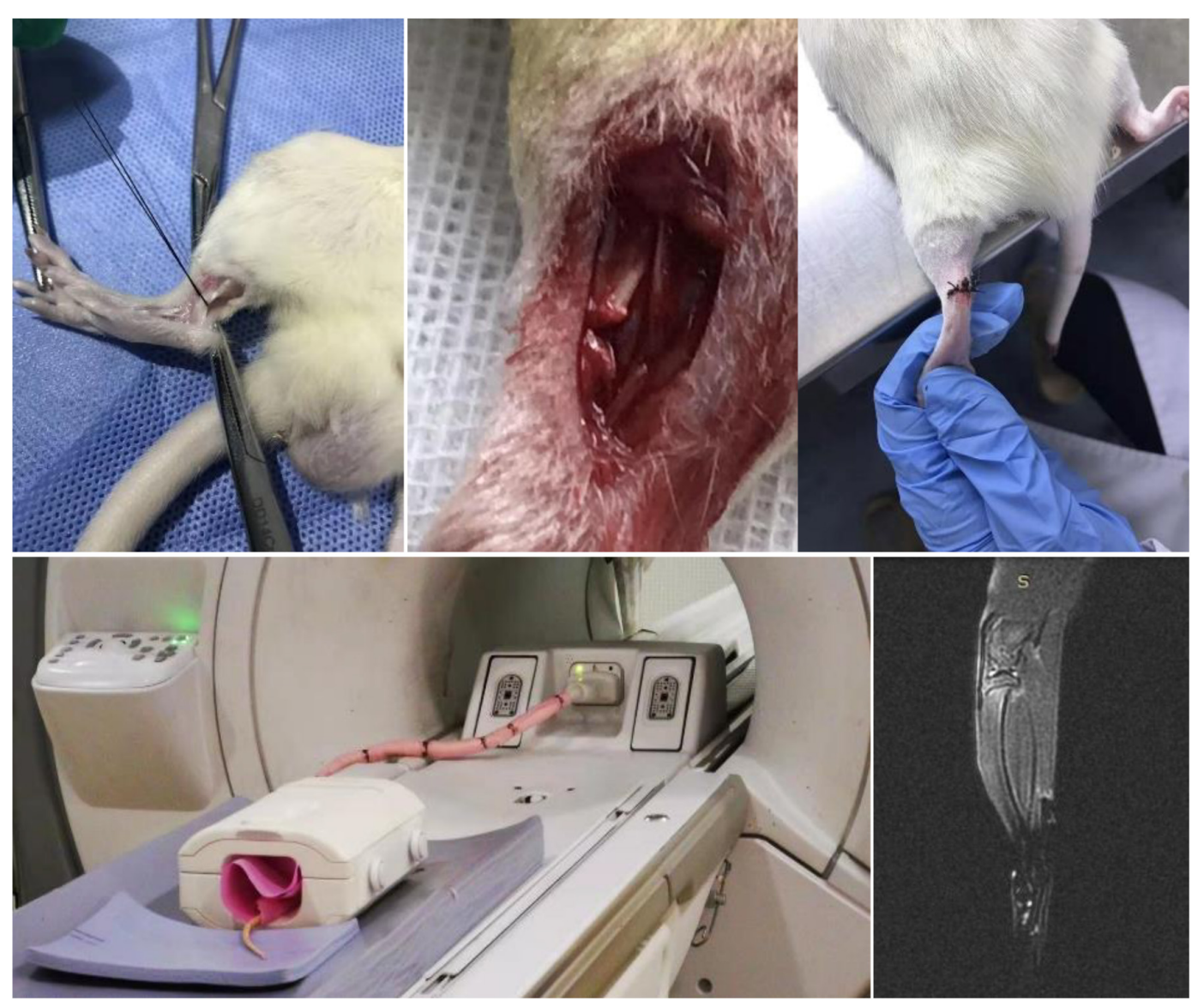

FIGURE 5 | MRI image of rupture and repair of Achilles tendon. Under the aseptic conditions, Achilles tendon of rats were performed with line transverse incision and skin incision with the careful protection of blood vessels to prevent damage after vascular cavity, obtaining the Achilles tendon rupture model with 1-2 mm long transection of the defect. The successful modeling was determined by MRI image.

for 1-day, Figure $\mathbf{4 A}$ showed that the cell viability showed over $95 \%$ of cell viability even after 3 days of culture, demonstrating good biocompatibility. Then, Cell viability was intuitively observed using a live/dead staining measurement. The extraction of gelatin/ $\mathrm{Fe}_{3} \mathrm{O}_{4} /$ celecoxib hydrogels in DMEM medium were added directly to the MC3T3-E1 cells and DAPI dyes. After incubation for $24 \mathrm{~h}$, they emitted the green fluorescents with healthy morphology (Figure 4B), revealing the excellent in vitro biocompatibility. Therefore, these gelatin/ $\mathrm{Fe}_{3} \mathrm{O}_{4} /$ celecoxib hydrogels could be utilized as potential candidates in various bio-applications.

\section{Animal Model of Tendon Tissue Injury}

To better understand the basic cellular and molecular biology of tendon repair, gelatin $/ \mathrm{Fe}_{3} \mathrm{O}_{4} /$ celecoxib hydrogels were performed to assess the repair efficiency on the Achilles tendon of adult rats, and changes in macrophage phenotypes and related genes were analyzed. Compared with uninjured controls, injury tendon tissues were collected after 3, 7, 14, and 28 days, and the results found the accumulation of M1 macrophages in the early stage and then the transformation into the temporal and spatial distribution of M2 phenotype in Figure 5.
It is known that change in the phenotype of macrophages play an important role in the tendon repair, and M2 macrophages mainly exists in the reshaping of the late stage. After treatment for $3,7,14$, and 28 days for the injured tendon tissue in rats, immunofluorescence technology test was investigated for evaluation of combination effects for tendon damage using M2 macrophages (CD163 antibody markers). The gelatin $/ \mathrm{Fe}_{3} \mathrm{O}_{4} /$ celecoxib sample showed that the number of M2 macrophages in all of groups were increased from the treatment of first 3 days, and achieve the highest proportion of M2 macrophages in the 2 weeks. In contrast, gelatin/ $/ \mathrm{Fe}_{3} \mathrm{O}_{4}$ and gelatin/celecoxib groups exhibited similar increase trends, which were higher than the untreated control (Figure 6). The results suggested that the combined treatments could promote the increase in the proportion of M2 macrophages, thus accelerating the tendon repair process.

In addition, we also evaluated the combination therapy via the inflammatory infiltration and pathological injury of tendon injury site. Clinical studies found that inflammation existed in the process of tendon injury repair, and tendon healing was a complex and long process, including inflammation stage, proliferation stage and remodeling stage. H\&E staining 


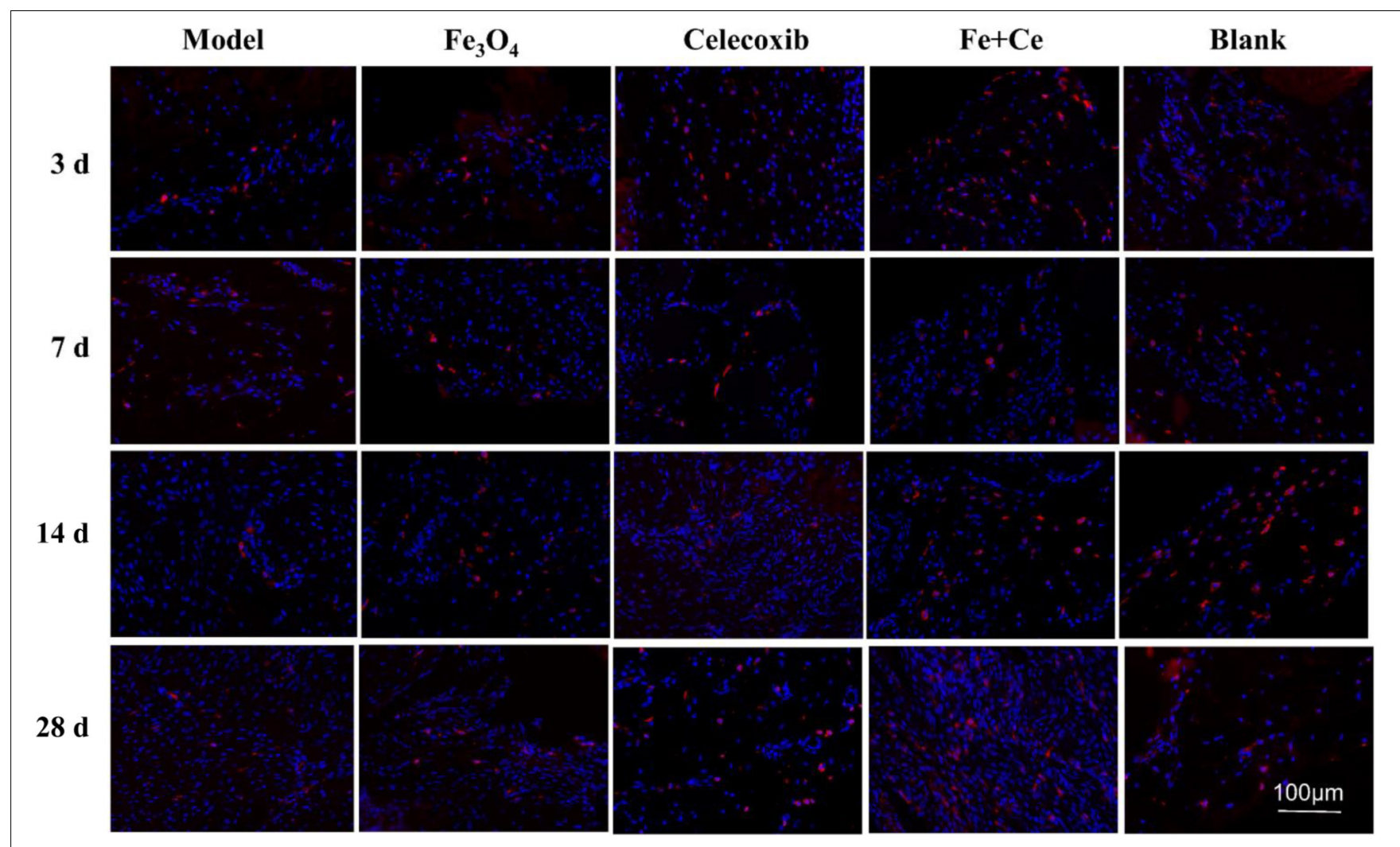

FIGURE 6 | The spatiotemporal changes of M2 macrophages in the tendon tissues. Red dots represent M2 macrophages. After treatment for 3 days, 1, 2, 4 weeks, IF test showed the M2 macrophages with CD163 antibody markers (red) changed. All of the experimental groups appeared the red fluorescence from the 3-day and continue to 28 days, wherein the combined treatment group exhibited the highest proportion of M2 macrophages.

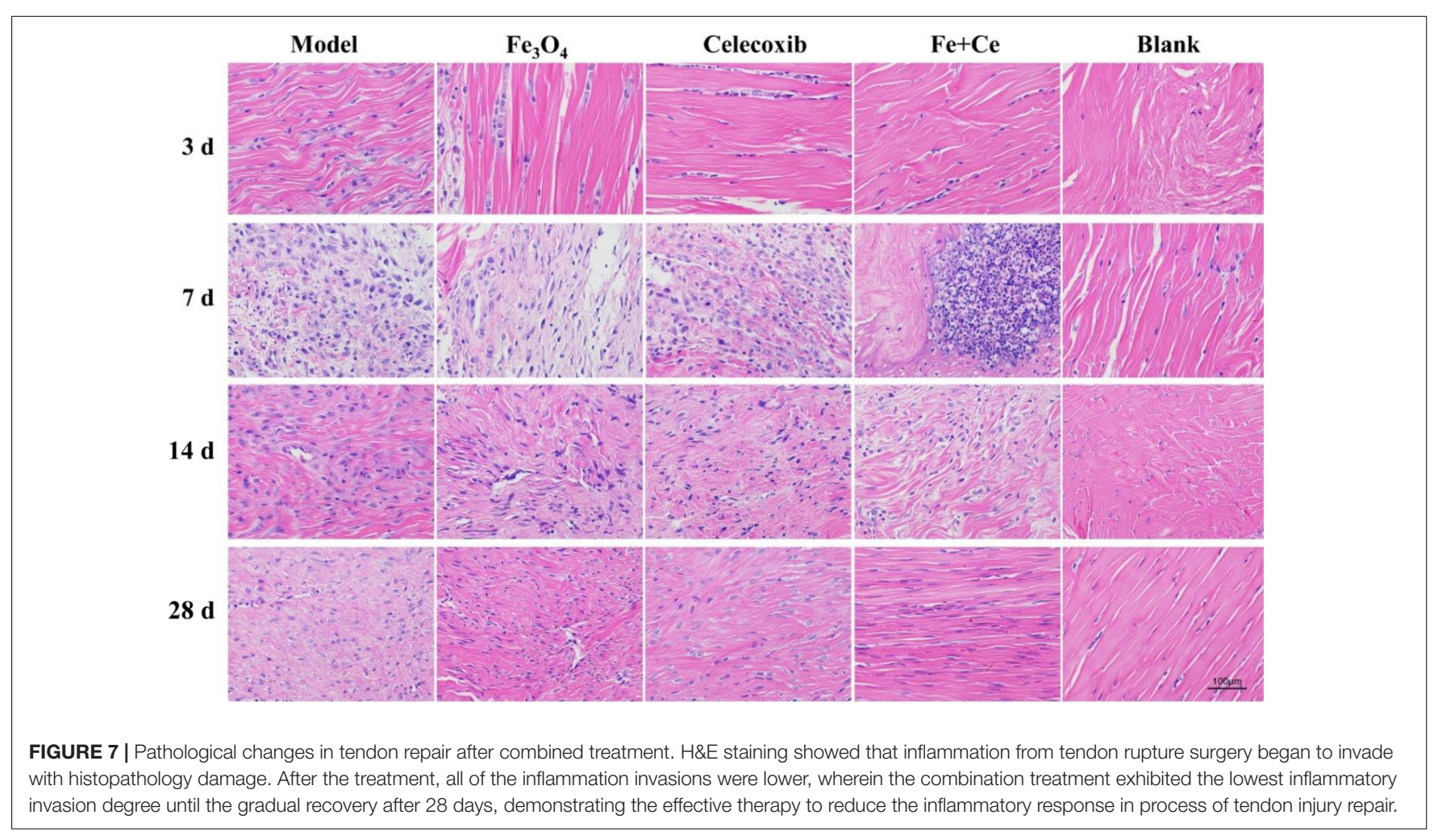


results showed that the control group exhibited serious tendon inflammation and histopathologic damage after the fracture operation invasion. After treatment for 3, 7, 14, and 28 days, gelatin $/ \mathrm{Fe}_{3} \mathrm{O}_{4}$, gelatin/celecoxib and gelatin $/ \mathrm{Fe}_{3} \mathrm{O}_{4} /$ celecoxib groups could reduce the inflammation invasion, wherein the synergistic therapy showed the least inflammation invasion as shown in Figure 7. These results demonstrated that synergistic treatment can effectively reduce the inflammatory response in the process of tendon injuries repair.

\section{Gait Analysis Using Catwalk}

The utility of gait analysis using CatWalk has been shown in various rodent models, such as sciatic nerve injury (Deumens et al., 2007), spinal cord injury (Hamers et al., 2006; Koopmans et al., 2005), myofascial inflammation (Miyagi et al., 2011), intervertebral disc injury (Miyagi et al., 2013), and inflammatory arthritis models (Ferreira-Gomes et al., 2008; Gabriel et al., 2007). It is well-known that injuries of limb joints and muscle tendons, tumors, malformations, neurological diseases and even psychological and mental states of the rats can affect the normal gait with various degrees. After the tendon injuries, the model rats can cause the long-term abnormal gait that may cause the secondary tendons damage and pains. Thus, the purpose of gait analysis in the present study was to identify the mechanism and causes of gait abnormalities, and treadmill running provide a platform and approach for gait analysis, which can obtain the quantitative and accurate gait data to provide the best treatment details and assess the rehabilitation efficacy. CatWalk gait analysis system, including the relevant parameters of standing phase time, swing phase time, footprints area, mean intensity and swing speed, can reflect the level of pain in rats and evaluate their tendon recovery, which provided important reference and indicators for the treatment and prognosis of tendon injuries after clinical trials. Therefore, we used the realtime Catwalk gait to analyze the tendon injury repair of the rats in Figure 8A.

As shown in Figures $\mathbf{8 B}-\mathbf{F}$, there were no significant differences in the standing phase time, swing phase time, footprints area, mean intensity and swing speed among all of the control group, model group, gelatin $/ \mathrm{Fe}_{3} \mathrm{O}_{4}$ group, gelatin/celecoxib group and gelatin $/ \mathrm{Fe}_{3} \mathrm{O}_{4} /$ celecoxib group after the postoperative treatment for 1 and 3 days. After the operation for 7 days, the standing time, swing phase time, footprints area, mean intensity and swing speed of all of
A

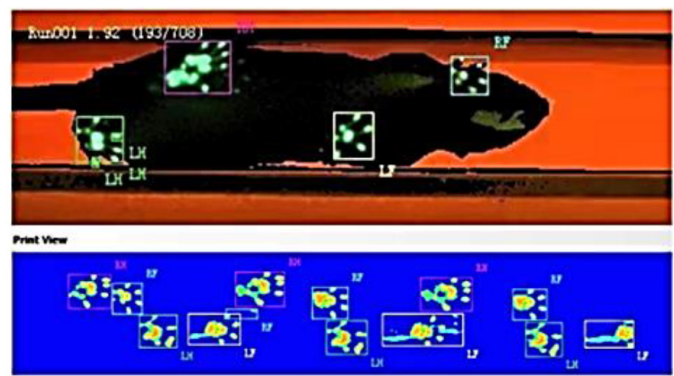

C

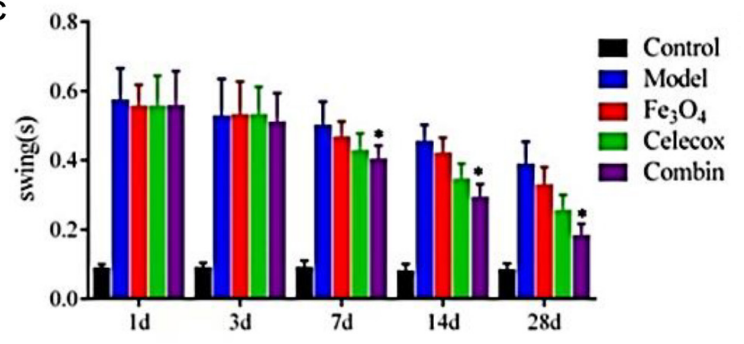

E

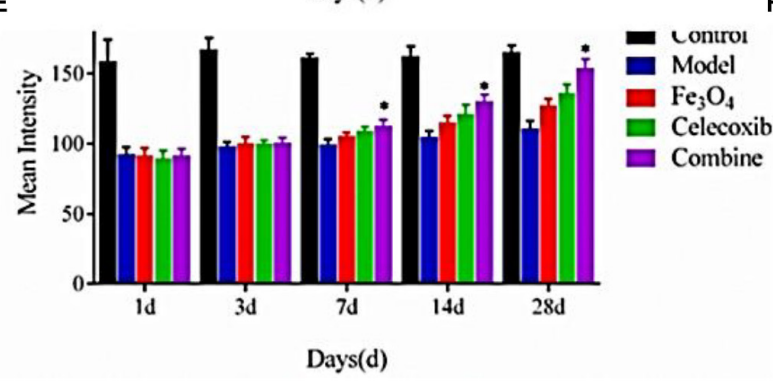

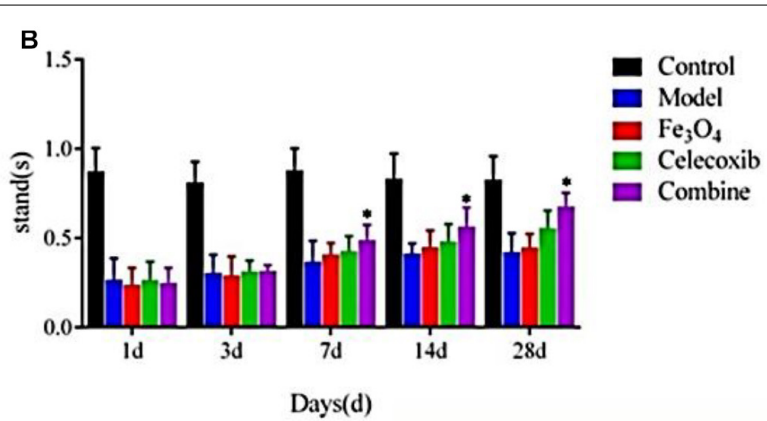

D

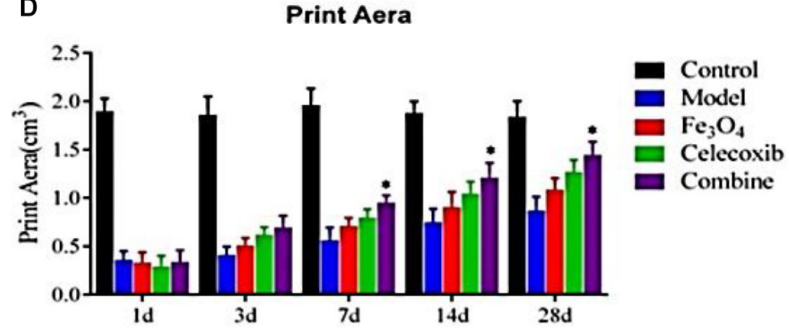

Days(d)

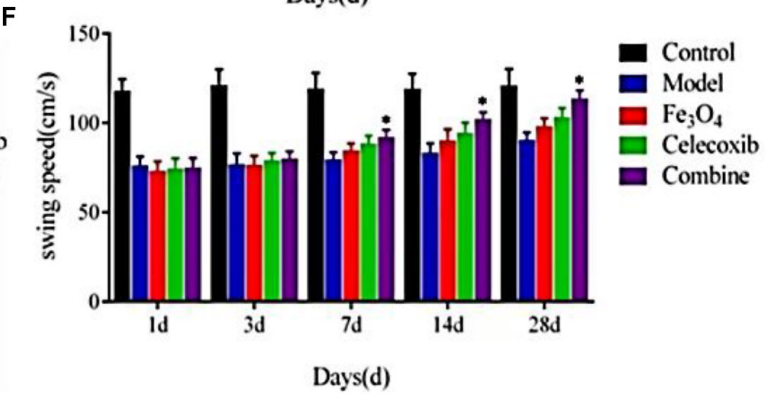

FIGURE 8 | (A) CatWalk gait analysis of tendon injury repair of the rats. (B) Standing phase time, (C) swing phase time, (D) footprints area, (E) mean intensity, and (F) swing speed of the repaired rats $\left({ }^{*} P<0.05\right)$. 
groups gradually increased. Until 28 days, the differences in the gelatin/ $\mathrm{Fe}_{3} \mathrm{O}_{4} /$ celecoxib group were statistically significant compared to other groups, indicating important roles in relieving the pain and promoting recovery prognosis. It was mentioned that gelatin/ $\mathrm{Fe}_{3} \mathrm{O}_{4}$ and gelatin/celecoxib groups exhibited similar therapy effects, demonstrating the effects of pulsed electromagnetic field therapy on Achilles tendon rupture injury, which also further indicated the therapeutic combination of pulsed electromagnetic field and drug possessed great potentials in the rehabilitation of Achilles tendon rupture that can hopefully improve the walking function and promote tendon repair for the patients.

\section{CONCLUSION}

In summary, we developed a new type of hydrogel dressing of gelatin $/ \mathrm{Fe}_{3} \mathrm{O}_{4} /$ celecoxib with combination therapies of celecoxib drug and pulse electromagnetic field for the injured tendon repair. The in vitro experiment revealed this gelatin $/ \mathrm{Fe}_{3} \mathrm{O}_{4} /$ celecoxib composite hydrogel system possessed functional characteristics and coordinated release behaviors under the external environmental stimuli. The in vivo evaluation showed a favorable repair by this synergistic strategy than that of single pulse electromagnetic or celecoxib drug, which not only effectively reduced the inflammatory reaction of macrophage cells but also contributed to the incremental proportion of M2 macrophages at the injury site. Furthermore, gait analysis revealed that synergistic therapy was important and effective to tendon injury repair in the later stage, that is, M2 macrophages played an active role in the production stage, and the polarization of M2-type macrophages was closely related to the repair of tendon injury. Therefore, this new type of combined tendon repair treatment will not only provide beneficially theoretical basis and therapeutic regimen for the clinical applications,

\section{REFERENCES}

Bao, W. R., Li, M. L., Yang, Y. Y., Wan, Y., Wang, X., Bi, N., et al. (2020). Advancements and frontiers in high performance of natural hydrogels for cartilage tissue engineering. Front. Chem. 8:53. doi: 10.3389/fchem.2020.00053

Biressi, S., Miyabara, E. H., Gopinath, S. D., Carlig, P. M., and Rando, T. A. (2014). A Wnt-TGF $\beta 2$ axis induces a fibrogenic program in muscle stem cells from dystrophic mice. Sci. Transl. Med. 6, 267-276. doi: 10.1126/scitranslmed. 3008411

Carr, A. J., Cooper, C. D., Campbell, M. K., Rees, J. L., Moser, J., Beard, D. J., et al. (2015). Clinical effectiveness and cost effectiveness of open and arthroscopic rotator cuff repair (the UK Rotator Cuff Surgery (UKUFF) randomised trial. Health Technol. Assess. 19, 1-218. doi: 10.3310/hta19800

Chen, Y. R., Zhou, Z. X., Zhang, J. Y., Yuan, F. Z., Xu, B. B., Guan, J., et al. (2019). Low-molecular-weight heparin-functionalized chitosan-chondroitin sulfate hydrogels for controlled release of TGF- $\beta 3$ and in vitro neocartilage formation. Front. Chem. 7:745. doi: 10.3389/fchem.2019.00745

Dakin, S. G., Martinez, F. O., Yapp, C., Wells, G., Oppermann, U., Dean, B. J., et al. (2015). Inflammation activation and resolution in human tendon disease. Sci. Transl. Med. 7, 311-373. doi: 10.1126/scitranslmed.aac4269

Deumens, R., Jaken, R. J., Marcus, M. A. E., and Joosten, E. A. J. (2007). The CatWalk gait analysis in assessment of both dynamic and static gait changes after adult rat sciatic nerve resection. J. Neurosci. Methods 164, 120-130. doi: 10.1016/j.jneumeth.2007.04.009 but also reduce the economic burden and social pressure for the patients.

\section{DATA AVAILABILITY STATEMENT}

The data that supports the plots within this paper and other findings of this study are available from the corresponding authors upon reasonable requests.

\section{ETHICS STATEMENT}

The animal study was reviewed and approved by Animal Ethics Committee of the Chinese PLA General Hospital. Written informed consent was obtained from the owners for the participation of their animals in this study.

\section{AUTHOR CONTRIBUTIONS}

LZ and YL initiated and designed the project, made suggestions, and revised the article. JW and LW collected the information and wrote this manuscript. YZ, YG, XH, TH, and BL checked and finalized the manuscript. All authors reviewed, commented, and approved the final manuscript.

\section{FUNDING}

This work was supported by the Provincial and Ministerial Science and Technology Joint Project (LHGJ20191056 and 2018020778), Education Department of Henan Province Project (WJLX2019322) and Henan Administration of Traditional Chinese Medicine Project (2019ZY3011).

Duffield, J. S. (2010). Macrophages and immunologic inflammation of the kidney. Semin. Nephrol. 30, 234-254. doi: 10.1016/j.semnephrol.2010.03.003

Ferreira-Gomes, J., Adaes, S., and Castro-Lopes, J. M. (2008). Assessment of movement-evoked pain in osteoarthritis by the knee-bend and CatWalk tests: a clinically relevant study. J. Pain 9, 945-954. doi: 10.1016/j.jpain.2008. 05.012

Gabriel, A. F., Marcus, M. A. E., Honig, W. M. M., Walenkamp, G. H. I. M., and Joosten, E. A. J. (2007). The CatWalk method: a detailed analysis of behavioral changes after acute inflammatory pain in the rat. J. Neurosci. Methods 163, 9-16. doi: 10.1016/j.jneumeth.2007.02.003

Global Burden of Disease Study 2013 Collaborators (2015). Global, regional, and national incidence, prevalence, and years lived with disability for 301 acute and chronic diseases and injuries in 188 countries, 1990-2013: a systematic analysis for the Global Burden of Disease Study 2013. Lancet 386, 743-800.

Gombozhapova, A., Rogovskaya, Y., Shurupov, V., Rebenkova, M., Kzhyshkowska, J., Popov, S. V., et al. (2017). Macrophage activation and polarization in postinfarction cardiac remodeling. Biomed. Sci. 24:13. doi: 10.1186/s12929-0170322-3

Hackett, L., Millar, N. L., Lam, P., and Murrell, G. A. (2016). Are the symptoms of calcific tendinitis due to neoinnervation and/or neovascularization? J. Bone Joint. Surg. Am. 98, 186-192. doi: 10.2106/jbjs.o.00417

Hamers, F. P., Koopmans, G. C., and Joosten, E. A. (2006). CatWalk-assisted gait analysis in the assessment of spinal cord injury. J. Neurotrauma 23, 537-548. doi: $10.1089 /$ neu.2006.23.537 
Koopmans, G. C., Deumens, R., Honig, W. M. M., Hamers, F. P. T., Steinbusch, H. W. M., and Joosten, E. A. J. (2005). The assessment of locomotor function in spinal cord injured rats: the importance of objective analysis of coordination. J. Neurotrauma 22, 214-225. doi: 10.1089/neu.2005.2 2.214

Li, D. W., Zhou, J., Zhang, M. M., Ma, Y. Z., Yang, Y. Y., Han, X., et al. (2020). Longterm delivery of alendronate through injectable tetra-PEG hydrogel to promote osteoporosis therapy. Biomater. Sci. 8, 3138-3146. doi: 10.1039/d0bm0 $0376 \mathrm{j}$

Linderman, S. W., Gelberman, R. H., Thomopoulos, S., and Shen, H. (2016). Cell and biologic-based treatment of flexor tendon injuries. Oper. Tech. Orthop. 26, 206-215. doi: 10.1053/j.oto.2016.06.011

Liu, Z. Y., Liu, J. H., Cui, X., Wang, X., Zhang, L. C., and Tang, P. F. (2020). Recent advances on magnetic sensitive hydrogels in tissue engineering. Front. Chem. 8:124. doi: 10.3389/fchem.2020.00124

Liu, H. Y., Wang, X., Cao, Y. X., Yang, Y. Y., Yang, Y. T., Gao, Y. F., et al. (2020). Freezing-tolerant, highly sensitive strain and pressure sensors assembled from ionic conductive hydrogels with dynamic cross-links. ACS Appl. Mater. Interf. 12, 25334-25344. doi: 10.1021/acsami.0c06067

Liu, B. C., Zhao, Y. R., Zhu, T. J., Gao, S., Ye, K. F., Zhou, F., et al. (2020). Biphasic double-network hydrogel with compartmentalized loading of bioactive glass for osteochondral defect repair. Front. Bioeng. Biotechnol. 8:752. doi: 10.3389/fbioe. 2020.00752

Martinez, F. O., and Gordon, S. (2014). The M1 and M2 paradigm of macrophage activation: time for reassessment. F1000Prime Rep. 6:13. doi: 10.12703/ P6-13

Miyagi, M., Ishikawa, T., Kamoda, H., Orita, S., Kuniyoshi, K., Ochiai, N., et al. (2011). Assessment of gait in a rat model of myofascial inflammation using the CatWalk system. Spine 36, 1760-1764. doi: 10.1097/brs.0b013e318226 9732

Miyagi, M., Ishikawa, T., Kamoda, H., Suzuki, M., Sakuma, Y., Orita, S., et al. (2013). Assessment of pain behavior in a rat model of intervertebral disk injury using the CatWalk gait analysis system. Spine 38, 1459-1465. doi: 10.1097/brs. 0b013e318299536a

Sugg, K. B., Lubardic, J., Gumucio, J. P., and Mendias, C. L. (2014). Changes in macrophage phenotype and induction of epithelial-to-mesenchymal transition genes following acute Achilles tenotomy and repair. J. Orthop. Res. 32, 944-951. doi: $10.1002 /$ jor.22624
Tang, G. K., Zhou, B. Y., Li, F., Wang, W. H., Liu, Y., Wang, X., et al. (2020). Advances of naturally-derived and synthetic hydrogels for intervertebral disc regeneration. Front. Bioeng. Biotechnol. 8:745. doi: 10.3389/fbioe.2020.00745

Tang, Q. M., Chen, J. L., Shen, W. L., Yin, Z., Liu, H. H., Fang, Z., et al. (2014). and adult fibroblasts display intrinsic differences in tendon tissue engineering and regeneration. Sci. Rep. 4:5515.

Voleti, P. B., Buckley, M. R., and Soslowsky, L. J. (2012). Tendon healing: repair and regeneration. Annu. Rev. Biomed. Eng. 14, 47-71.

Wang, X., Yang, Y. Y., Shi, Y., and Jia, F. (2020). Editorial: smart hydrogels in tissue engineering and regenerative medicine. Front. Chem. 8:245. doi: 10.3389/fchem. 2020.00245

Wanitphakdeedecha, R., Sathaworawong, A., Manuskiatti, W., and Sadick, N. S. (2017). Efficacy of multipolar radiofrequency with pulsed magnetic field therapy for the treatment of abdominal cellulite. J. Cosmet. Laser. Ther. 19, 205-209. doi: 10.1080/14764172.2017.1279332

Xu, S. J., Yang, F., Zhou, X., Zhuang, Y. P., Liu, B. X., Mu, Y., et al. (2015). Uniform PEGylated PLGA microcapsules with embedded Fe3O4 nanoparticles for US/MR dual-modality imaging. ACS Appl. Mater. Interf. 7, 20460-20468. doi: 10.1021/acsami.5b06594

Yang, Y. Y., Yang, Y. T., Cao, Y. X., Wang, X., Chen, Y. R., Liu, H. Y., et al. (2021). Anti-Freezing, Resilient and tough hydrogels for sensitive and largerange strain and pressure sensors. Chem. Eng. J. 403:126431. doi: 10.1016/j.cej. 2020.126431

Zhang, L. N., Zuo, X. Q., Li, S. J., Sun, M., Xie, H. M., Zhang, K., et al. (2019). Synergistic therapy of magnetism-responsive hydrogel for soft tissue injuries. Bioact. Mater. 4, 160-166. doi: 10.1016/j.bioactmat.2019.03.002

Conflict of Interest: The authors declare that the research was conducted in the absence of any commercial or financial relationships that could be construed as a potential conflict of interest.

Copyright (c) 2020 Wang, Wang, Gao, Zhang, Huang, Han, Liu, Zhang, Li and Zhang. This is an open-access article distributed under the terms of the Creative Commons Attribution License (CC BY). The use, distribution or reproduction in other forums is permitted, provided the original author(s) and the copyright owner(s) are credited and that the original publication in this journal is cited, in accordance with accepted academic practice. No use, distribution or reproduction is permitted which does not comply with these terms. 